\title{
Aesthetic evaluation of the labiolingual position of maxillary lateral incisors by orthodontists and laypersons
}

Xue Jiang ${ }^{1,4+}$, Zhiwei $\mathrm{Cao}^{1+}{ }^{1+}$ Yang Yao $^{2}$, Zhihe Zhao ${ }^{3}$ and Wen Liao ${ }^{3^{*}}$

\begin{abstract}
Background: The maxillary anterior teeth play a crucial role in smile aesthetics. Previous studies regarding the importance of maxillary lateral incisors for smile aesthetics concentrated on their size, incisor edge level, and inclination, etc. However, the aesthetic effect of lateral incisor movement in the spatial position has not been studied yet. Therefore, the purpose of this study was to explore the influence of the labiolingual position of maxillary lateral incisors on the aesthetic perception of smiles by orthodontists and laypersons, as well as analyze differences in this perception between male and female raters.

Methods: A three-dimensional (3D) dental model was generated from the photograph of a man's smile using iOrtho7.0 software (Time Angel, Wuxi, China). Based on this model, seven images were generated with different labiolingual positions of the maxillary lateral incisors in $0.5 \mathrm{~mm}$ increments (+ indicating labial translation, and -indicating lingual translation). The images were evaluated by 86 orthodontists and 161 laypersons using a visual analog scale, with lower scores indicating less attractiveness. Data were analyzed using Student's $t$ test and one-way analysis of variance with post hoc test.
\end{abstract}

Results: There was no significant difference in smile ratings by males and females. Orthodontists assigned lower scores to all images than laypersons. The smile at $+1.5 \mathrm{~mm}$ was considered the least attractive by orthodontists, while smiles at $+1.5 \mathrm{~mm}$ and $-1.5 \mathrm{~mm}$ were regarded as the least attractive by laypersons. The smile at $0 \mathrm{~mm}$ was evaluated as the most attractive by all raters. Laypersons gave different scores to smiles at 0 or $-0.5 \mathrm{~mm}$, but orthodontists did not.

Conclusions: The labiolingual position of maxillary lateral incisors does affect the perception of smile aesthetics. Orthodontists may rate smile aesthetics more critically than laypersons. Therefore, communication and discussion between orthodontists and patients is needed to achieve better therapeutic and aesthetic outcomes.

Keywords: Aesthetic evaluation, Smile, Maxillary lateral incisors, Orthodontists, Laypersons

*Correspondence: liaowenssw@126.com

${ }^{\dagger}$ Xue Jiang and Zhiwei Cao contributed equally to this work

${ }^{3}$ State Key Laboratory of Oral Diseases and National Clinical Research

Center for Oral Diseases, Department of Orthodontics, West China

Hospital of Stomatology, 3rd section of Renmin South Road,

Chengdu 610041, Sichuan Province, China

Full list of author information is available at the end of the article

\section{Background}

The goal of dental treatment is not only to restore normal functions, but also to endow patients with certain aesthetics. The concept of beauty is unquantifiable because it is influenced by many factors, such as different cultures and beliefs. Many studies have demonstrated that orthodontists and laypersons have different perceptions of 
smile aesthetics, and that orthodontists are more sensitive to deviations from the ideal [1-3].

The maxillary anterior teeth play a crucial role in smile aesthetics [4]. A majority of studies regarding maxillary lateral incisors have concentrated on their size [1], incisor edge level [5], inclination [6], and replacement of lateral incisors with canines [7]. However, the aesthetic effect of lateral incisor movement in the spatial position has not been studied yet.

Dental crowding is one of the most common types of dental malocclusion. One of the consequences of dental crowding is the spatial displacement of teeth. Previous studies have simulated the rotational displacement of the maxillary lateral incisors and central incisors due to crowded dentition $[8,9]$. Labiolingual movement of maxillary lateral incisors is also a common deformity caused by crowded dentition, yet we are unaware of studies about the aesthetic effects of such movement.

Therefore, this study was designed to determine the effect of labial-palatal movement of maxillary lateral incisors on the aesthetic perception of smiles by orthodontists and laypersons. We also assessed whether the rater's sex influenced his or her perception.

\section{Methods}

\section{Acquisition and processing of images}

The study received ethical approval from the Ethics Committee of the West China School of Stomatology from Sichuan University (WCHSIRB-D-2019-066). A male volunteer aged 20 who had not received any orthodontic or conservative/prosthetic treatment was selected as a model. His smile was considered highly attractive according to the following principles: symmetry of maxillary central incisors, gingival display of less than $1.0 \mathrm{~mm}$, and proper smile arc $[10,11]$. The volunteer's smile was photographed using a Canon EOS 7D under standard conditions, and the brightness, contrast, and midline tilt of the photo were adjusted using Adobe Photoshop (CC2018, Adobe Systems, San Jose, CA, USA). The upper twothirds of the face were removed to minimize interference [12].

Digital three-dimensional (3D) models of maxillary and mandibular dentitions of the model's smile were generated using Sirona D3492 (Sirona Dental Systems GmbH, Bensheim, Germany). The software iOrtho7.0 (Time Angel, Wuxi, China) was used to alter the position of teeth in the 3D model. The original position of maxillary lateral incisors was considered the control image $(0 \mathrm{~mm})$. In the occlusal view, all teeth were aligned in a harmonious archform (Fig. 1). The left lateral incisors were shifted to different labiolingual positions via translation in the 3D models obtained using iOtho7.0, and then a screenshot of the front view of the entire model was taken. The

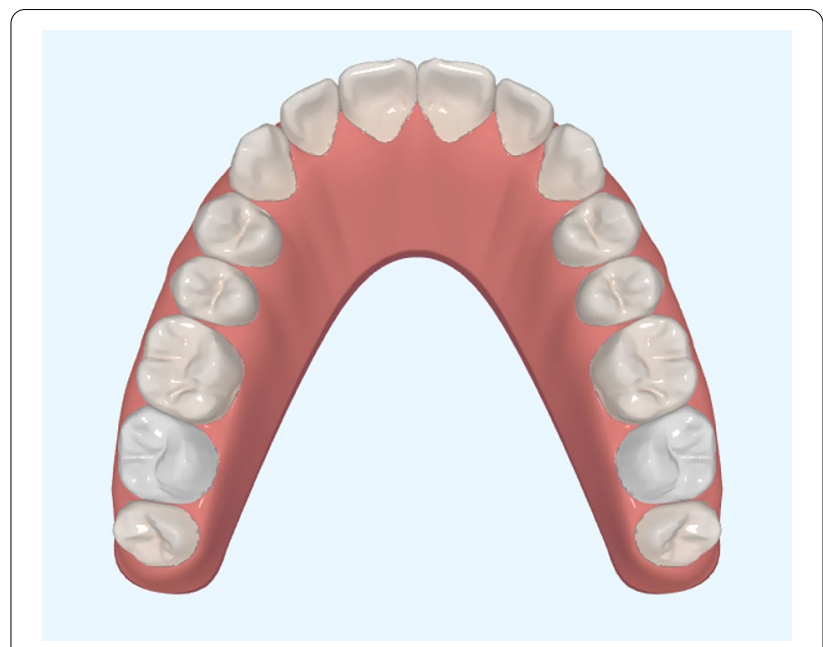

Fig. 1 Three-dimensional digital simulation of maxillary dentition (occlusal view)

screenshots of the digital simulations were used as references to change the left lateral incisor position in the twodimensional (2D) photograph using Adobe Photoshop. Through this method, the volunteer's left maxillary lateral incisors were shifted in the 3D models and 2D photos by $-1.5 \mathrm{~mm},-1 \mathrm{~mm},-0.5 \mathrm{~mm}, 0 \mathrm{~mm},+0.5 \mathrm{~mm}$, $+1 \mathrm{~mm}$, or $+1.5 \mathrm{~mm}$. The minus sign indicated lingual movement; the plus sign, labial movement. The "0 $\mathrm{mm}$ " meant that the lateral incisor had not moved from a reasonable position relative to the central incisor; that is to say, at $0 \mathrm{~mm}$, the occlusal view showed a smooth curve of incisors. A mirror transformation was applied in order to generate a right-side image from the left side and thereby eliminate aesthetic interference caused by asymmetry.

In this manner, both a frontal view (Fig. 2) and an occlusal view (Fig. 3) were obtained of the digital 3D models and 2D photos (Fig. 4). The occlusal view of the $3 \mathrm{D}$ model served to indicate how the modifications had been performed. The modified $2 \mathrm{D}$ photos were used in the questionnaire shown to the evaluators.

\section{Selection of participants}

Two groups of people were selected as evaluators of the photos, orthodontists and laypersons. Pilot study data from 18 participants in each group was calculated using PASS software (version 11.0; NCSS, USA) for to verify the sample size. Based on a level of significance of $5 \%$ $(\alpha=0.05)$ and $80 \%$ power, the sample size was calculated. The results indicated that 28 raters in each group were needed.

To ensure even larger samples than this minimum, we invited 93 orthodontists and 245 laypersons, aged between 18 and 50 years, to participate in the study. 

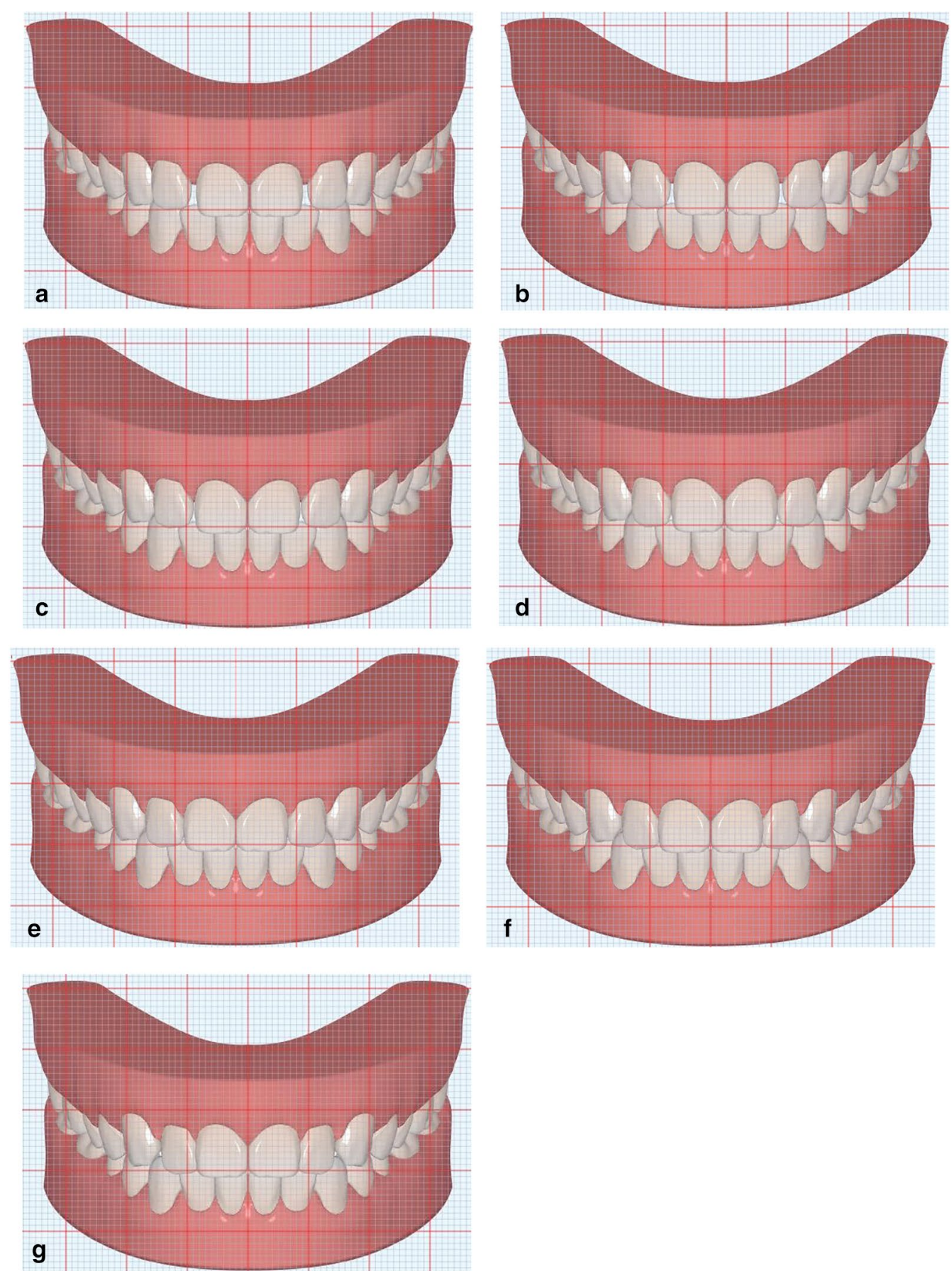

Fig. 2 Images of three-dimensional digital simulations were used as references (frontal view). a $+1.5 \mathrm{~mm}$, labial movement with $1.5 \mathrm{~mm}$. b+ $1.0 \mathrm{~mm}$, labial movement with $1.0 \mathrm{~mm}$. c $+0.5 \mathrm{~mm}$, labial movement with $0.5 \mathrm{~mm}$. d $0 \mathrm{~mm}$, control group. e $-0.5 \mathrm{~mm}$, lingual movement with $0.5 \mathrm{~mm}$. $\mathbf{f}-1.0 \mathrm{~mm}$, lingual movement with $1.0 \mathrm{~mm} . \mathbf{g}-1.5 \mathrm{~mm}$, lingual movement with $1.5 \mathrm{~mm}$

Raters were excluded from the study if they could not identify any difference between any of the photos or had difficulty in understanding the questionnaires.

\section{Questionnaire}

SurveyStar (Changsha, China) was used to create the questionnaire, which was distributed to the evaluators and answered online. Seven 2D photos were presented in random order. Participants were asked to score the 
a
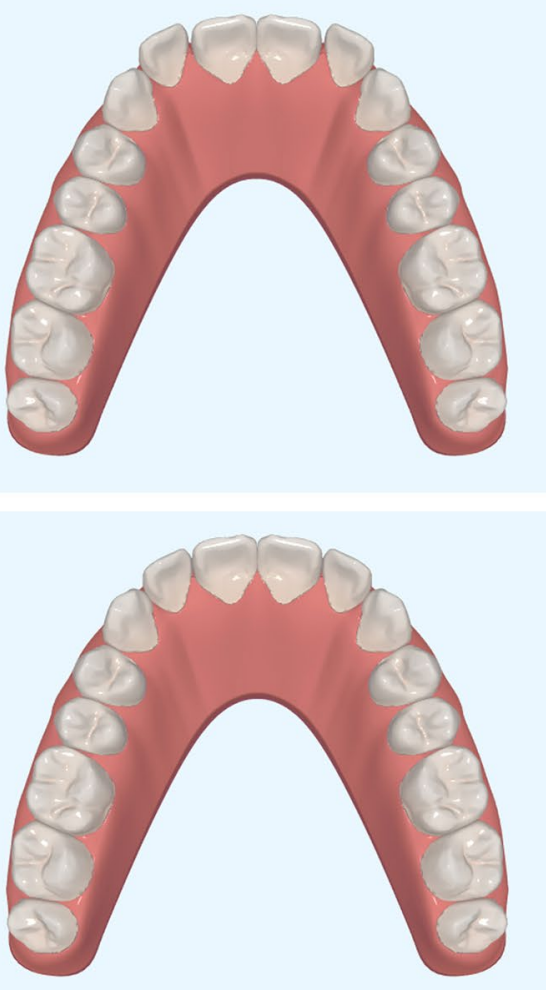

C

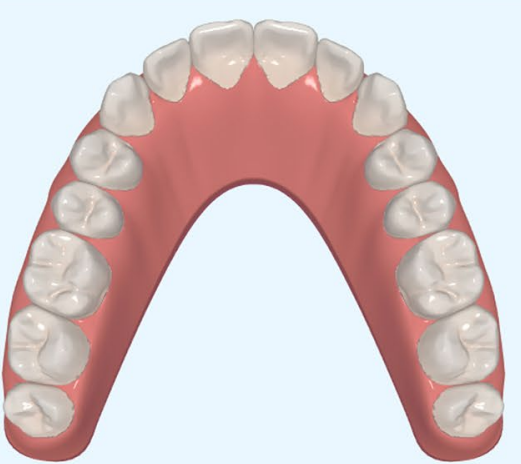

e

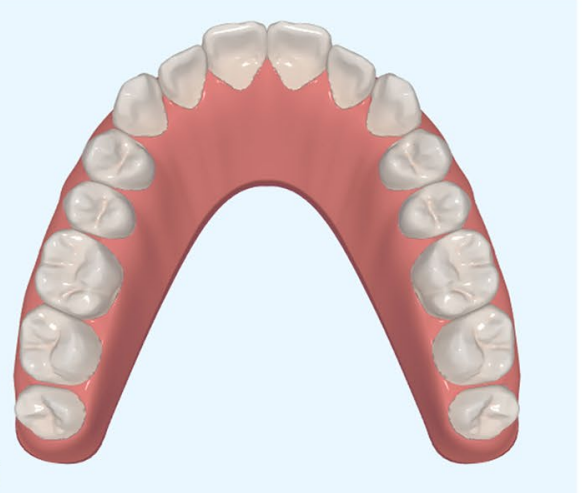

b

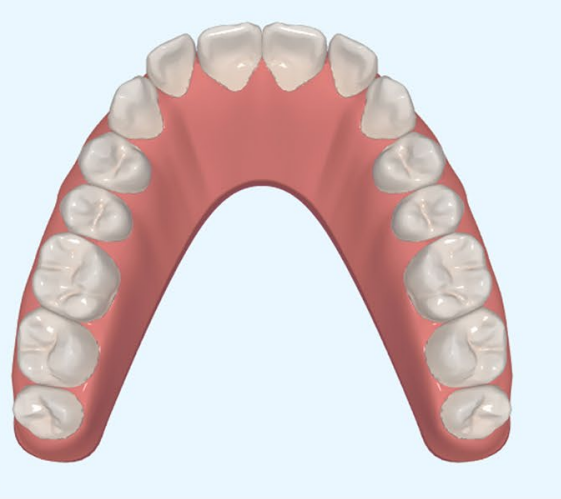

d
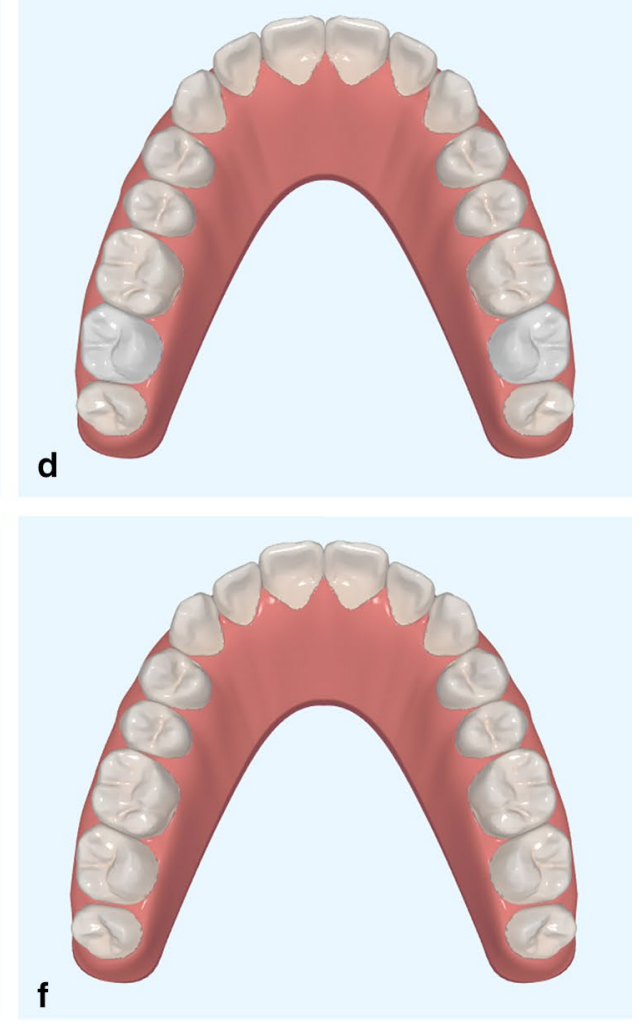

ces (occlusal view). $\mathbf{a}+1.5 \mathrm{~mm}$, labial movement with $1.5 \mathrm{~mm}$

Fig. 3 Images of three-dimensional digital simulations were used as references (occlusal view). a + $1.5 \mathrm{~mm}$,labial movement with $1.5 \mathrm{~mm}$. b $+1.0 \mathrm{~mm}$, labial movement with $1.0 \mathrm{~mm}$. c $+0.5 \mathrm{~mm}$, labial movement with $0.5 \mathrm{~m}$ $0.5 \mathrm{~mm}$. $\mathbf{f}-1.0 \mathrm{~mm}$, lingual movement with $1.0 \mathrm{~mm} . \mathbf{g}-1.5 \mathrm{~mm}$, lingual movement with $1.5 \mathrm{~mm}$ 

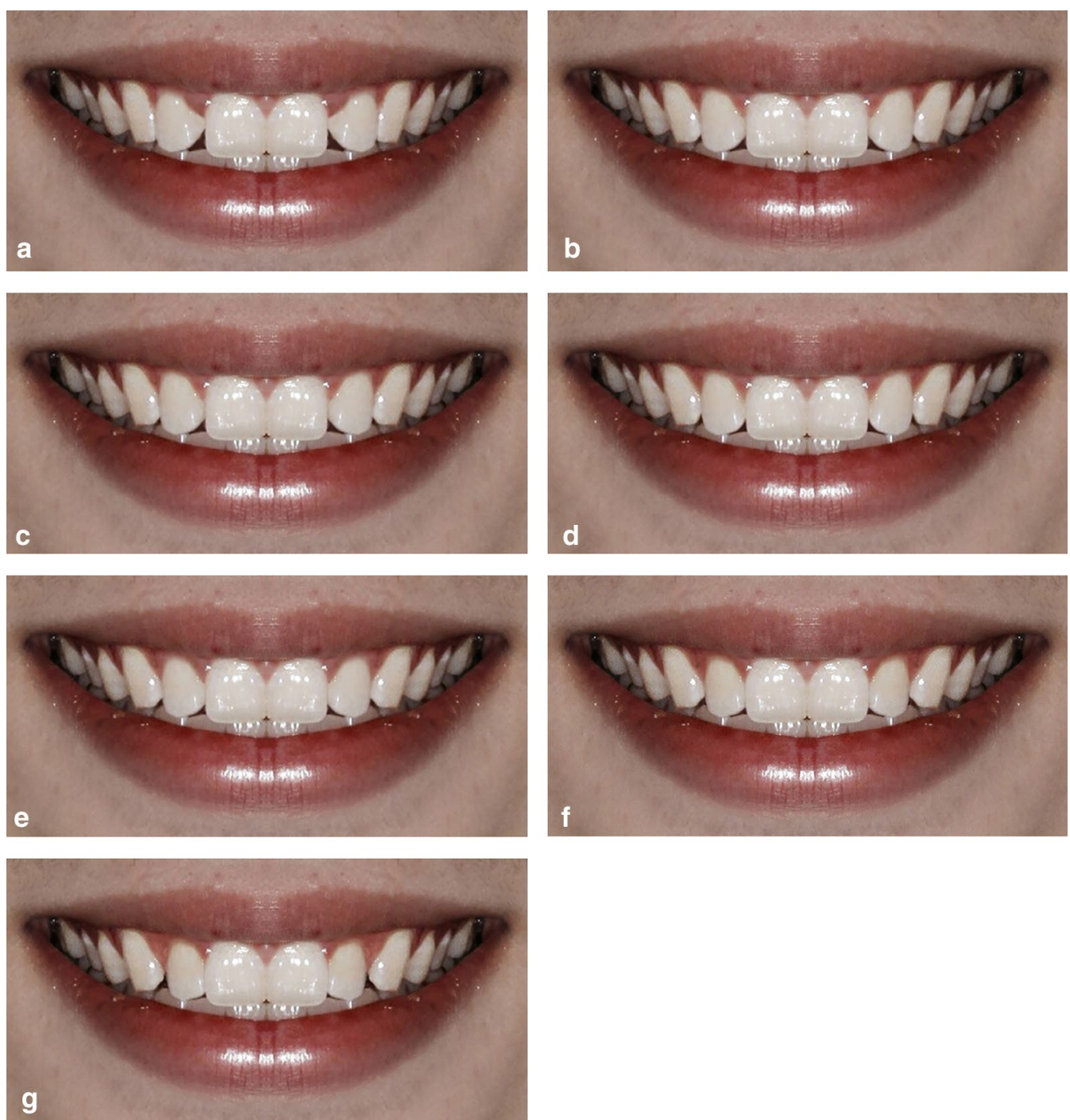

Fig. 4 The labiolingual position of the maxillary lateral incisor was changed in $0.5 \mathrm{~mm}$ increments. a $+1.5 \mathrm{~mm}$,labial movement with $1.5 \mathrm{~mm}$. b+ $1.0 \mathrm{~mm}$, labial movement with $1.0 \mathrm{~mm}$. c $+0.5 \mathrm{~mm}$, labial movement with $0.5 \mathrm{~mm}$. d $0 \mathrm{~mm}$, control group. e $-0.5 \mathrm{~mm}$, lingual movement with $0.5 \mathrm{~mm}$. $\mathbf{f}-1.0 \mathrm{~mm}$, lingual movement with $1.0 \mathrm{~mm} . \mathbf{g}-1.5 \mathrm{~mm}$, lingual movement with $1.5 \mathrm{~mm}$

photos by gliding a slider depicting a visual analogue scale (VAS) below the images. The rightmost end of the slider indicated most attractive (100 points); the leftmost end of the slider indicated least attractive (0 points). Each photo was displayed only once and could not be rescored.

\section{Reliability}

To determine the reliability of our results, a random subset of 33 participants were asked two weeks later to reassess the same seven images. The earlier and later scores for photos showed an intraclass correlation coefficient of 0.91 for orthodontists and 0.98 for laypersons [13], indicating good reliability for both groups.

\section{Statistical analysis}

All data were recorded in Microsoft Excel (Microsoft Office 2010, Microsoft Corporation, USA) and then analyzed using SPSS 23.0 (IBM, Armonk, NY, USA). To assess the significance of differences in the mean VAS scores across seven images, one-way ANOVA with the post hoc test was used. Student's $t$ test was used to analyze differences between the scores of male and female raters for each image, or between the scores of orthodontists and laypersons. The level of significance was determined at the $5 \%$ level $(P \leq 0.05)$. 
Table 1 Characteristics of the raters who evaluated smile aesthetics

\begin{tabular}{lll}
\hline Characteristic & $\begin{array}{l}\text { Orthodontists } \\
(\mathbf{n = 8 6 )}\end{array}$ & Laypersons $(\mathbf{n = 1 6 1 )}$ \\
\hline Sex & & \\
Male & $22(25.58)$ & $35(21.74)$ \\
Female & $64(74.42)$ & $126(78.26)$ \\
Age group & & \\
18-25 years & $21(24.42)$ & $130(80.75)$ \\
26-30 years & $23(26.74)$ & $17(10.56)$ \\
$31-40$ years & $26(30.23)$ & $11(6.83)$ \\
$41-50$ years & $16(18.60)$ & $3(1.86)$ \\
\hline
\end{tabular}

Values are $n(\%)$

Table 2 Comparison of scoring by male or female raters* for different labiolingual positions of maxillary lateral incisors

\begin{tabular}{llll}
\hline Image & Male $(\mathbf{n}=\mathbf{5 7})$ & Female $(\mathbf{n = 1 9 0 )}$ & $\boldsymbol{P}_{\text {-value }}{ }^{* *}$ \\
\hline $\mathrm{A}(+1.5 \mathrm{~mm})$ & $34.61(20.31)$ & $37.33(23.23)$ & 0.427 \\
$\mathrm{~B}(+1.0 \mathrm{~mm})$ & $48.07(22.22)$ & $47.39(24.00)$ & 0.849 \\
$\mathrm{C}(+0.5 \mathrm{~mm})$ & $64.65(23.59)$ & $66.37(21.82)$ & 0.609 \\
$\mathrm{D}(0 \mathrm{~mm})$ & $74.32(17.00)$ & $75.48(18.71)$ & 0.673 \\
E $(-0.5 \mathrm{~mm})$ & $68.33(19.44)$ & $67.94(20.57)$ & 0.899 \\
F $(-1.0 \mathrm{~mm})$ & $64.00(20.26)$ & $69.70(21.47)$ & 0.076 \\
$\mathrm{G}(-1.5 \mathrm{~mm})$ & $44.33(20.58)$ & $44.71(23.23)$ & 0.911 \\
\hline
\end{tabular}

Values are mean (SD), unless otherwise noted

* Orthodontists and laypersons combined

** Student's $t$ test

\section{Results}

Among the 247 raters aged between 18 and 50, 86 were orthodontists and 161 were laypersons. Among orthodontists, $22(25.58 \%)$ were male and $64(74.42 \%)$ were female; among the laypersons, 35 (21.74\%) were male and 126 (78.26\%) were female (Table 1$)$. There was no statistical difference between males and females in VAS scoring of each image (Table 2).

The average scores for each image given by orthodontists and laypersons are shown in Table 3. From the perspective of orthodontists, image A was considered to be the least attractive smile (mean score of 31.69), while the most attractive was image D (mean score of 70.74). Among the laypersons, the lowest scores were assigned to images A (mean score 39.39) and G (mean score 46.16), while image $\mathrm{D}$ was also rated as the most attractive (mean score 77.60).

The scores for images A, B, C, and D differed significantly between orthodontists and laypersons, whereas they did not for images E, F, or G. The scores awarded by orthodontists were lower than those awarded by laypersons for the same image, indicating that the orthodontists were stricter in evaluating the smile aesthetics.

\section{Discussion}

Previous work $[14,15]$ has shown that the anterior teeth play an important role in smile aesthetics. Previous studies of lateral incisors indicated that the width ratio of lateral incisors to central incisors should correspond to the golden ratio $[1,16]$. However, many studies later reported that the golden ratio of lateral incisors to central incisors has negligible effect on smile aesthetics [17]. For example, one work [1] showed that lateral incisors with a width of $67-72 \%$ of the central incisors and a length $1.5 \mathrm{~mm}$ shorter than central incisors were considered attractive. Another study [18] demonstrated that most people preferred short, broad lateral incisors, and they could tolerate a small mesial inclination of lateral incisors. A third study [6] compared different aesthetic changes due to different teeth inclinations and suggested

Table 3 Scoring of each image by orthodontists or laypersons

\begin{tabular}{|c|c|c|c|c|c|}
\hline Image & $\begin{array}{l}\text { Orthodontists }(n=86) \\
\text { Mean (SD) }\end{array}$ & Results* & $\begin{array}{l}\text { Laypersons }(\mathrm{n}=161) \\
\text { Mean (SD) }\end{array}$ & Results* & $P$-value** \\
\hline $\mathrm{A}(+1.5 \mathrm{~mm})$ & $31.69(21.27)$ & 1 & $39.39(22.86)$ & i & 0.010 \\
\hline $\mathrm{B}(+1.0 \mathrm{~mm})$ & $42.37(22.39)$ & $\|$ & $50.31(23.76)$ & $\mathrm{ii}$ & 0.011 \\
\hline$C(+0.5 \mathrm{~mm})$ & $59.79(21.78)$ & III & $69.27(21.79)$ & iii & 0.001 \\
\hline $\mathrm{D}(0 \mathrm{~mm})$ & 70.74 (19.67) & IV & 77.60 (17.12) & iv & 0.005 \\
\hline $\mathrm{E}(-0.5 \mathrm{~mm})$ & $66.31(21.79)$ & III,IV & $68.95(19.43)$ & iii & 0.331 \\
\hline $\mathrm{F}(-1.0 \mathrm{~mm})$ & $67.28(20.41)$ & III,IV & $68.98(21.79)$ & iii & 0.552 \\
\hline $\mathrm{G}(-1.5 \mathrm{~mm})$ & $41.77(23.40)$ & $\|$ & $46.16(22.09)$ & $\mathrm{i}, \mathrm{ii}$ & 0.146 \\
\hline
\end{tabular}

$\mathrm{SD}$, standard deviation

* Comparison of labiolingual position of maxillary lateral incisor within groups by one-way ANOVA and post hoc test. There were no significant differences between the same Roman numerals variables

${ }^{* *}$ Comparison between orthodontists and laypersons using Student's $t$ test 
that slight symmetrical mesial inclination of both lateral incisors could make a smile more attractive.

The studies mentioned above included only tooth changes in a 2D plane, but inclination and displacement of teeth in 3D planes are quite common in orthodontic cases. One study [19] studied different anteroposterior positions of the maxillary central incisors, showing that raters were more tolerant of labial protrusion of the maxillary central incisors than of lingual retrusion. Another study [20] found that labial inclination and lingual retrusion of maxillary central incisors were relatively unacceptable. A third study [21] examined the labiolingual inclination and anteroposterior position of maxillary incisors in three different facial patterns, showing that different facial patterns led to different aesthetic criterion; nevertheless, consistently with previous studies, raters showed less tolerance for the labiolingual movement of maxillary central incisors than for their labiolingual inclination.

The Little's Irregularity Index (LII), the sum of the distances between the four anterior teeth's anatomic contact points, is an important index of dentition crowding [22]. However, studies have suggested that the LII index lacks reproducibility [23, 24]. Scanning models may be more reliable than the LII index [23]. In the present study, the Sirona intra-oral scanner was used to obtain the volunteer's digital dentition models. and iOrtho7.0 software was used to change the position of maxillary lateral incisors. The 2D photos were created based on the visual effect of the 3D models, so that the changes taking place in 3D direction were transformed into 2D images. As orthodontists face more cases of 3D malformation of teeth or dentition in the clinics, our research method may provide references for future research. The angle used in taking the photographs should be matched with the angle to view the dental cast in iOrtho7.0 software, which should be explored in future research.

Our study also confirmed that VAS can be used for the analysis of various factors affecting smile aesthetics due to its convenience and repeatability [25]. Some studies asked participants to rank photos in the order of attractiveness to find which photo was the most or the least attractive [14]. Other studies combined ranking orders and VAS scores together and evaluated their consistency [13]. We did not ask the raters to rank the photos in order of preference, since it seemed likely that greater amounts of labial or lingual movement would be judged correspondingly less acceptable.

The rater's sex may be one of the factors influencing perception of smile aesthetics [18], but some studies [1, 26] have shown that there are no significant differences between male and female raters, which is consistent with our study. Studies have shown that the sex of the subject whose smile is under evaluation could also influence the perceived smile aesthetics [27, 28]. In our study, only one photograph of a front view of a man's smile was used, and only the part of the mouth was retained to minimize the effects of the subject's gender. Therefore, future studies are needed to assess potential differences in how a man or woman's smile aesthetics depends on the labiolingual position of the maxillary lateral incisors. Future work should also explore perception of smile aesthetics based on lateral or 3/4-lateral views, and not only the frontal view in the present study. Such work should also examine how smile aesthetics change when more than one tooth movement is involved, which is the more frequent situation in the clinic.

Our study indicated that orthodontists and laypersons awarded the highest score to the control group $(0 \mathrm{~mm})$, and they gave lower scores as the moving distance of maxillary lateral incisors increased. Compared with laypersons, orthodontists had a lower tolerance for labial movement of lateral incisors, and they assigned marginally lower scores when there was lingual movement of maxillary lateral incisors. This indicates that orthodontists had higher aesthetic standards, and were more sensitive than laypersons when the lateral incisors were moved labially. Laypersons and orthodontists alike were less tolerant of labial movement than lingual movement over the same distance. This is in contrast with a study [20], which reported the lingual movement and labial inclination of the maxillary central incisors were less acceptable. The divergence of the conclusions may result from the fact that different labiolingual position of the maxillary central incisors tends to change the position of the entire dentition, and the receding dentition could make the smile look less full [19], and people value fuller smiles. Our study was based on the position of the maxillary lateral incisors, which changed the position independently of the entire dentition. Moreover, a front-view picture was used in our research instead of a profile picture as in that previous study [20], which can also explain the different observations.

In our study, orthodontists did not find a difference between smiles at $0 \mathrm{~mm}$ and $-0.5 \mathrm{~mm}$, while laypersons did. This was contrary to previous work where orthodontists were more likely to distinguish between subtle changes in dentition [1-3]. Therefore, before orthodontic treatment, communication and discussion between orthodontists and patients is needed to achieve better therapeutic and aesthetic outcomes.

Our study presents several limitations. First, we found that, when we changed the position of lateral incisors in the 3D model after fixing the adjacent teeth, the space between the lateral incisor and its adjacent teeth was magnified on the photographs of $+1.0 \mathrm{~mm}$ and $+1.5 \mathrm{~mm}$, 
which was somewhat different from the actual situation. In future studies, we will seek a better balance between controlling a single variable and simulating the clinical situation, which may make the results more useful. Second, the effect of rater age on their scoring was not considered when designing this study, so we did not recruit sufficient, similar numbers of raters across relevant age groups. Orthodontists are generally older than orthodontic patients, as was the case in our study, so future work should examine the potential influence of age on perception of smile aesthetics.

\section{Conclusions}

In our study, the sex of the evaluators did not seem to affect their scoring of smiles with different labiolingual positions of maxillary lateral incisors. The labial position of the maxillary lateral incisors was more unacceptable than that of lingual position to all raters, who agreed that the smile with unmoved maxillary lateral incisors $(0 \mathrm{~mm})$ was the most attractive. Orthodontists were stricter than laypersons with respect to the labial position of maxillary lateral incisors, while there was no significant difference between their scoring in the lingual position. Hence, before orthodontic treatment, communication and discussion between orthodontists and patients is needed to achieve better therapeutic and aesthetic outcomes.

\section{Abbreviations}

LII: Little's Irregularity Index; VAS: Visual analogue scale; 2D: Two-dimensional; 3D: Three-dimensional.

\section{Acknowledgements}

The authors would like to thank the volunteers who took part in this study, and they are grateful to Dr. R. Scott Conley for kindly proofreading this manuscript and giving valuable suggestions.

\section{Authors' contributions}

All authors contributed to this article. XJ and ZC collected the data, analyzed the data and drafted the manuscript. WL designed and guided the experimental work. YY and ZZ revised the manuscript. All authors read and agreed with the content of the submitted manuscript.

\section{Funding}

This work was supported by the Science and Technology Department of Sichuan Province (2020YFS0087) and the Sichuan University-Luzhou City Cooperation Program (2018CDLZ-14).

\section{Availability of data and materials}

The datasets used and analyzed during the current study are available from the corresponding author on reasonable request.

\section{Ethics approval and consent to participate}

The data collected from the questionnaire does not contain any private information of the participants and written informed consent for publication was obtained from the volunteer who provided his smile photographs in the questionnaire and article. All of our work was approved by the Ethics Committee of the West China School of Stomatology from Sichuan University (WCHSIRB-D-2019-066) and applicable patient release was obtained. Relevant documents can be found in the supplementary material.

\section{Consent to publish}

Written informed consent for publication was obtained from the volunteer who provided his smile photographs in the questionnaire and article.

\section{Competing interests}

We declare no potential conflicts of interest with respect to the authorship or publication of this manuscript.

\section{Author details}

${ }^{1}$ State Key Laboratory of Oral Diseases and National Clinical Research Center for Oral Diseases, West China School of Stomatology, Sichuan University, Chengdu, China. ${ }^{2}$ State Key Laboratory of Oral Diseases and National Clinical Research Center for Oral Diseases, Department of Implantology, West China Hospital of Stomatology, Sichuan University, Chengdu, China. ${ }^{3}$ State Key Laboratory of Oral Diseases and National Clinical Research Center for Oral Diseases, Department of Orthodontics, West China Hospital of Stomatology, 3rd section of Renmin South Road, Chengdu 610041, Sichuan Province, China ${ }^{4}$ Department of Implant Dentistry, National Clinical Research Center for Oral Diseases, Shanghai Key Laboratory of Stomatology, Shanghai Ninth People's Hospital, School of Medicine, Shanghai Jiao Tong University, Shanghai, China.

Received: 26 April 2020 Accepted: 17 January 2021

Published online: 22 January 2021

\section{References}

1. Bukhary SMN, Gill DS, Tredwin CJ, Moles DR. The influence of varying maxillary lateral incisor dimensions on perceived smile aesthetics. Br Dent J. 2007;203(12):687-93.

2. Jarosz KF, Bosio JA, Bloomstein R, Jiang SS, Vakharia NS, Cangialosi TJ. Perceptions of chin asymmetries among dental professionals and laypersons. Am J Orthod Dentofac Orthop. 2018;154(2):201-12.

3. Pinho S, Ciriaco CJ, Lenza M. Impact of dental asymmetries on the perception of smile esthetics. Am J Orthod Dentofac Orthop. 2007;132(6):748-53

4. Machado AW. 10 commandments of smile esthetics. Dent Press J Orthod. 2014;19(4):136-57.

5. King KL, Evans CA, Viana G, BeGole E, Obrez A. Preferences for vertical position of the maxillary lateral incisors. World J Orthod. 2008;9(2):147-54.

6. Brunzel S, Kern M, Freitag S, Wolfart S. Aesthetic effect of minor changes in incisor angulation: an internet evaluation. J Oral Rehabil. 2006;33(6):430-5.

7. Brough E, Donaldson AN, Naini FB. Canine substitution for missing maxillary lateral incisors: the influence of canine morphology, size, and shade on perceptions of smile attractiveness. Am J Orthod Dentofac Orthop. 2010;138(6):701-5.

8. Alqahtani N, Preston B, Guan G. Perception of dental professionals and lay persons to altered mandibular incisors crowding. J World Fed Orthod. 2012;1(2):e61-5.

9. Ma W, Preston B, Asai Y, Guan H, Guan G. Perceptions of dental professionals and laypeople to altered maxillary incisor crowding. Am J Orthod Dentofac Orthop. 2014;146(5):579-86.

10. Machado AW, Moon W, Gandini LJ. Influence of maxillary incisor edge asymmetries on the perception of smile esthetics among orthodontists and laypersons. Am J Orthod Dentofac Orthop. 2013;143(5):658-64.

11. Ribeiro JB, Figueiredo BA, Machado AW. Does the presence of unilateral maxillary incisor edge asymmetries influence the perception of smile esthetics? J Esthet Restor Dent. 2017;29(4):291-7.

12. De-Marchi LM, Pini NI, Ramos AL, Pascotto RC. Smile attractiveness of patients treated for congenitally missing maxillary lateral incisors as rated by dentists, laypersons, and the patients themselves. J Prosthet Dent. 2014;112(3):540-6.

13. Yüksel AG, Iskender SY, Kuitert R, Papadopoulou AK, Dalci K, Darendeliler MA, et al. Differences in attractiveness comparing female profile modifications of Class II Division 1 malocclusion. Am J Orthod Dentofac Orthop. 2017;152(4):471-6.

14. Hussain A, Louca C, Leung A, Sharma P. The influence of varying maxillary incisor shape on perceived smile aesthetics. J Dent. 2016;50:12-20.

15. Ong E, Playle RA, Richmond S. Peer assessment of dental attractiveness. Am J Orthod Dentofac Orthop. 2006;130(2):163-9. 
16. Carter NE, Gillgrass TJ, Hobson RS, Jepson N, Eechan JG, Nohl FS, Nunn JH. The interdisciplinary management of hypodontia: orthodontics. Br Dent J. 2003;194(7):361-6.

17. Sarver DM. Principles of cosmetic dentistry in orthodontics: Part 1. Shape and proportionality of anterior teeth. Am J Orthod Dentofac Orthop. 2004;126(6):749-53.

18. Pani SC, Fernandez RA, Kudsi Rl, Elkhawly FF, Albatran AN. The impact of lateral incisor dimensions on smile perception-a study of patients tolerance for deviation from the ideal. Periodontics Prosthodont. 2016:2:1.

19. Schlosser JB, Preston CB, Lampasso J. The effects of computer-aided anteroposterior maxillary incisor movement on ratings of facial attractiveness. Am J Orthod Dentofac Orthop. 2005;127(1):17-24.

20. Cao L, Zhang K, Bai D, Jing Y, Tian Y, Guo Y. Effect of maxillary incisor labiolingual inclination and anteroposterior position on smiling profile esthetics. Angle Orthod. 2011;81(1):121-9.

21. Chirivella P, Singaraju GS, Mandava P, Reddy VK, Neravati JK, George SA. Comparison of the effect of labiolingual inclination and anteroposterior position of maxillary incisors on esthetic profile in three different facial patterns. J Orthod Sci. 2017;6(1):1-10.

22. Little RM. The irregularity index: a quantitative score of mandibular anterior alignment. Am J Orthod. 1975;68(5):554-63.

23. Macauley D, Garvey TM, Dowling AH, Fleming GJ. Using Little's Irregularity Index in orthodontics: outdated and inaccurate? J Dent. 2012;40(12):1127-33.
24. Sjögren APG, Lindgren JE, Huggare JÅV. Orthodontic study cast analysis-reproducibility of recordings and agreement between conventional and 3D virtual measurements. J Digit Imaging. 2010;23(4):482-92.

25. Oliveira PL, Motta AF, Guerra CJ, Mucha JN. Comparison of two scales for evaluation of smile and dental attractiveness. Dent Press J Orthod. 2015;20(2):42-8.

26. Pithon MM, Matos VO, Coqueiro RDS. Upper incisor exposure and aging: perceptions of aesthetics in three age groups. J World Fed Orthod. 2015;4(2):57-62.

27. Geron S, Atalia W. Influence of sex on the perception of oral and smile esthetics with different gingival display and incisal plane inclination. Angle Orthod. 2005;75(5):778-84.

28. Zhang YF, Xiao L, Li J, Peng YR, Zhao Z. Young people's esthetic perception of dental midline deviation. Angle Orthod. 2010;80(3):515-20.

\section{Publisher's Note}

Springer Nature remains neutral with regard to jurisdictional claims in published maps and institutional affiliations.
Ready to submit your research? Choose BMC and benefit from:

- fast, convenient online submission

- thorough peer review by experienced researchers in your field

- rapid publication on acceptance

- support for research data, including large and complex data types

- gold Open Access which fosters wider collaboration and increased citations

- maximum visibility for your research: over $100 \mathrm{M}$ website views per year

At BMC, research is always in progress.

Learn more biomedcentral.com/submissions 